\title{
Solvation shift of a conical intersection in clusters of excited tetrakis(dimethyl amino)ethylene with ammonia and acetonitrile molecules
}

\author{
S. Sorgues, J.-M. Mestdagh and B. Soep \\ Laboratoire Francis Perrin (CNRS-URA-2453), \\ DSM/DRECAM/Service des Photons, Atomes et Molécules, \\ C.E.A. Saclay, \\ F-91191 Gif-sur-Yvette cedex, France
}

\begin{abstract}
The supersonic expansion of the title molecule (TDMAE) with a mixture of helium and a solvent molecule $\mathrm{S}(\mathrm{S}=$ ammonia or acetonitrile), generates a beam carrying $\operatorname{TDAME}(\mathrm{S}) \gtrsim 10$ clusters. The femtosecond pump (266 nm)probe $(800 \mathrm{~nm})$ technique is used to investigate their excited state dynamics. It corresponds to a wavepacket movement along slopes of the $S_{1}$ potential energy surface of TDMAE where the molecule switches from the valence $\mathrm{V}\left(\pi \pi^{*}\right)$ to the Zwitterionic $\mathrm{C}^{+} \mathrm{C}^{-}$configuration. As a first approximation, this occurs in the vicinity of a conical intersection. The present results where TDMAE is solvated by polar molecules, are compared with former results using argon as a solvent. Whereas argon slows down of the wavepacket movement by what was called a chistera effect, the present case is dominated by a change in the location of the conical intersection that accelerates the time scale for the V-to-Z energy transfer. The interaction of a final charge transfer state with the solvent is also discussed.
\end{abstract}

Submitted to Chem. Phys. Lett.

Version date: September 6, 2004 


\section{Introduction}

Tetrakis(dimethyl amino)ethylene is an alkene-like molecule of structural formula<smiles>CN(C)C(=C(N(C)C)N(C)C)N(C)C</smiles>

Its real time dynamics has been investigated in the present laboratory following excitation to the $\mathrm{S}_{1}$ energy surface, either when the TDMAE molecule is free $[1,2]$ or solvated in an argon cluster [3, 4] or within a TDMAE cluster [5].

Attention has been drawn to the TDMAE molecule because it is a textbook example where the excited state dynamics involves a dramatic change of the electronic configuration that can be followed step-by-step. In these studies, a wavepacket is launched on the $\mathrm{S}_{1}$ surface with an electronic configuration corresponding to the $\pi \pi^{*}$ excitation of the central $\mathrm{CC}$ bond (the valence configuration $\mathrm{V}$, according to the terminology for alkene molecules [6]). Then, the wavepacket starts moving along the slopes of the $\mathrm{S}_{1}$ potential energy surface and reaches a region where the dominant electronic configuration is zwitterionic $(\mathrm{Z})$ with the character $\mathrm{C}^{+} \mathrm{C}^{-}$on the central $\mathrm{CC}$ bond. As a first approximation the switch from $\mathrm{V}$ to $\mathrm{Z}$ occurs at a conical intersection. Following the excitation of $\mathrm{V}$ at $266 \mathrm{~nm}$, the configuration switch is achieved in $300 \mathrm{fs}$ in free TDMAE [1]. More recent investigation in this laboratory indicate that the switch is mediated by an intermediate Rydberg configuration [work in progress].

The present work extends our former investigations where TDMAE is embedded into a cluster. In the present case, clusters of polar molecules, ammonia and acetonitrile, are considered. The aim is to unravel by which mechanism, the passage of the wavepacket in the CI region is affected by the presence of a medium surrounding the TDMAE molecule. The issue is of importance considering that the CI concept is now routinely invoked to rationalize the dynamics of molecules or of molecular groups that are embedded in solvents $[7,8]$ or proteins $[9,10]$. This issue is part of a more general concern that has been reviewed recently: the role of cluster solvation in reaction dynamics $[11,12]$. 


\section{Experimental}

The experimental apparatus is extensively described elsewhere [1, 4]. It associates a pulsed supersonic beam with a Time-Of-Flight Mass-Spectrometer (TOF-MS). It is coupled to the LUCA/SLIC femtosecond laser facility of Saclay to perform standard pump (266 nm)-probe(800 $\mathrm{nm})$ experiments.

The beam is generated from a mixture helium, ammonia (or acetonitrile) and TDMAE. A $5 \%$ mixture of either ammonia or acetonitrile with helium is passing over a TDMAE cell at room temperature before the supersonic expansion. This provides us with a helium-ammonia (or acetonitrile)-TDMAE mixture that is brought to expansion from a stagnation pressure $\mathrm{P}_{0}$ that can be varied from 1 to 7 bars. Mass spectra obtained by focusing the laser on the beam are shown in Figure (1) for the helium/ammonia/TDMAE expansion. The TDMAE molecule is considerably more condensable than ammonia. For this reason, the mass spectrum at low stagnation pressure (1 bar) is dominated by ions issued from TDMAE dimers, trimers or larger clusters. The partial pressure of TDMAE decreases as the stagnation pressure is increased and above $\mathrm{P}_{0}=5$ bars, it is small enough for TDMAE $\left(\mathrm{NH}_{3}\right)_{\mathrm{n}}$ clusters to dominate. The present work concentrates on this regime, hence documenting the dynamics of a single excited TDMAE molecule within ammonia (or acetonitrile) clusters. Because of fragmentation phenomena in the ionisation chamber of the TOF-MS, the size of the clusters actually present in the beam is certainly larger than that of the clusters observed in Figure (1). As we shall see below this is not an issue since the signals that are observed are not specific of the size of the cluster.

The pump laser at $266 \mathrm{~nm}$ performs a one photon excitation of the TDMAE molecule within the clusters and the probe laser at $800 \mathrm{~nm}$ achieves ionisation. The power density of the probe is lower than $2 \times 10^{11} \mathrm{~W} \mathrm{~cm}^{-2}$, enough to perform both one- and two-photon ionisation of the electronically excited TDMAE within the clusters. Thus allowing to detect both the $\mathrm{V}$ and $\mathrm{Z}$ configurations of TDMAE. The crosscorrelation width of the lasers is $110 \mathrm{fs}$. This excitation scheme is common with former 
experiments performed in this group $[1,4]$.

\section{$3 \quad$ Results and data analysis}

The signal measured at mass $234 \mathrm{amu}\left(\mathrm{TDMAE}\left(\mathrm{NH}_{3}\right)_{2}\right)$ in the ammonia experiment and that measured at mass $241 \mathrm{amu}$ in the acetonitrile experiment are shown in Figure (2) as a function of the $\operatorname{pump}(266 \mathrm{~nm})$ - $\operatorname{probe}(800 \mathrm{~nm})$ delay, up to a $1.2 \mathrm{ps}$ delay. Importantly, as in our former experiment on $\operatorname{TDMAE}(\mathrm{Ar})_{\mathrm{n}}$, evaporation phenomena are active and signals measured at a given ion mass do not reflect the behaviour of a neutral parent of the same mass, but the behaviour of parents carrying a larger number of solvent molecules [4]. As in reference [4] also, the signals shown in Figure (2) can be understood as resulting from a sequential 3 level scheme and can be fitted by the following expression after convoluting $\mathrm{S}(\mathrm{t})$ by the cross-correlation function of the lasers:

$$
\begin{aligned}
& S(t<0)=0 \\
& S(t \geq 0)=\sigma_{1} \exp \left(-\frac{t}{\tau_{1}}\right)+\sigma_{2} \frac{\tau_{2}}{\tau_{2}-\tau_{1}}\left[\exp \left(-\frac{t}{\tau_{2}}\right)-\exp \left(-\frac{t}{\tau_{1}}\right)\right] .
\end{aligned}
$$

The pump pulse, populates level 1 that decays at a rate $\mathrm{k}_{1}=\frac{1}{\tau_{1}}$ into level 2 which further decays into 3 at a rate $\mathrm{k}_{2}=\frac{1}{\tau_{2}} \cdot \sigma_{1}$ and $\sigma_{2}$ are the efficiencies for detecting levels 1 and 2. Level 3 is assumed to be non detectable. With the timescale of the figure, the second exponential appears as a plateau and only the time constant $\tau_{1}$ is documented by these fits, respectively $\tau_{1}=110 \pm 10$ and $150 \pm 20$ fs in the ammonia and acetonitrile experiments. Signal have been recorded at longer times also in the ammonia experiment. A time constant $\tau_{2}=100 \pm 10$ ps has been found.

\section{Discussion and conclusion}

The interpretation of Figure (2) follows that of our former works on the TDMAE dynamics and $\tau_{1}$ is assigned to the transition time when the wavepacket travels from the region of valence electronic configuration $(\mathrm{V})$ where it is detected by one-photon ionisation, to the zwitterionic region $\mathrm{Z}$ where it is detected by a two-photon ionisation process. The time constant $\tau_{2}$ corresponds to the lifetime of TDMAE when it has 
reached the $\mathrm{Z}$ configuration. When the TDMAE molecule is free, it corresponds to the transition time from $\mathrm{Z}$ to a charge transfer state that fluoresces to the ground state [1].

We focuss first on the time constant $\tau_{1}$. The present work complements other works performed in this laboratory and $\tau_{1}$ is documented now both for free TDMAE and when the molecule is embedded in various cluster environments. The available results are brought together in Table (1). The context of the discussion is that $\tau_{1}$ reflects the time constant of an internal conversion where the wavepacket movement in the excited potential energy surface allows electronic energy to be converted as vibrational energy. Two kinds of cluster effects can be anticipated in such a process. One is a simple perturbation of the wavepacket movement by the solvent, due to inertial effects that affect the molecular deformations. Broadly speaking, cage effects enter into this category. The other is more profound and corresponds to changing the arrangement of the potential energy surfaces involved in the wavepacket dynamics, hence changing the location of conical intersections between surfaces and more generally changing the coupling between potential surfaces.

The data of Table (1) are discussed now and the time constant $\tau_{1}$ measured for free TDMAE, $300 \mathrm{fs}$, serves as a reference. The solvating media that appear in the table fall in two categories : argon that increases $\tau_{1}$ from 300 to 400 fs and the other solvating species that reduce $\tau_{1}$ substantially. A link must be done immediately between these two categories of solvent, the intensity of the interaction between the solvent and excited TDMAE and in fine the two kinds of solvent effects that are anticipated above. This link serves as a guide in the following.

Argon, that increases $\tau_{1}$, has only a weak van der Waals interaction with TDMAE. The chistera effect, a variant to standard cage effects where inertia slows down the movement of the wavepacket has been proposed as responsible for the enhanced time constant in this case [4]. This is an effect of the first kind.

In contrast, the three species that reduce $\tau_{1}$ interact much more strongly with TDMAE. Let us recall first the interpretation of the TDMAE cluster experiment reported 
in Ref. [5]. In this case, TDMAE is its own solvent and electronic excitation may delocalise over several molecules. However local excitation of a single TDMAE molecule is assumed in this work and the 200 fs time constant is assigned to the transition from a $\mathrm{V}$ to a $\mathrm{Z}$ electronic configuration in a situation where the energy of these configurations are shifted by the solvation with the ground state TDMAE molecules that solvate the excited one. The diradicaloid V configuration was not expected to be strongly affected in the TDMAE cluster with respect to the monomer. In contrast, the zwitterionic configuration, with its charge separation character, was expected to be substantially stabilised by solvation. With the simple picture that the $\mathrm{V}$ to $\mathrm{Z}$ exchange occurs in the vicinity of a conical intersection, the latter is readily moved closer to the FrankCondon region of the pump excitation by solvation and thus is encountered at earlier times by the descending wavepacket. This effect was observed as unsensitive to the cluster size, indicating that one TDMAE molecules is sufficient to stabilise the Z state in clusters, a reasonable assumption considering that the TDMAE molecule with its 8 methyl substituents is extremely bulky, thus the addition of other units should impart little additional stabilisation to the $\mathrm{Z}$ state.

The same interpretation is transposed in the present work when TDMAE is solvated in ammonia and acetonitrile clusters. Both these molecules are polar with large permanent electric dipole moments of $1.47 \mathrm{D}$ for ammonia and $3.92 \mathrm{D}$ for acetonitrile [13]. They are expected to interact more strongly with excited TDMAE than ground state TDMAE in TDMAE clusters. Hence, the stabilisation the Z configuration of TDMAE should be larger when solvated by these molecules than in pure TDMAE clusters. As a result, the time constant $\tau_{1}$ should be somewhat smaller in the present work than the 200 fs reported in Ref. [5]. This is actually what observed in Table (1). However the ordering between acetonitrile and ammonia is surprising. The molecule with the largest dipole moment, acetonitrile, leads to a slightly larger time constant than ammonia (150 versus $110 \mathrm{fs}$ ). The fact that in the results reported in Figure (2) two ammonia molecules versus a single acetonitrile molecule are attached to 
the $\mathrm{TDMAE}^{+}$ion is not relevant to explain this result. First, the same time constants $\tau_{1}$ as those reported in this figure were observed for ions carrying more than 2 ammonia or more than one acetonitrile molecule. Second, because of fragmentation phenomena post to ionisation, several solvent molecules are lost by the cluster ion in the ionisation chamber of the TOF-MS. Hence, the ion signal reported in the figure reflect the behaviour of larger parents. The results of Table (1) thus indicate that the embedding of TDMAE by ammonia molecules produces a larger stabilization of the TDMAE(Z) configuration than the embedding by acetonitrile. This might be connected to the way solvating molecules are organized about the TDMAE molecule. Assuming that the interaction between TDMAE and either ammonia or acetonitrile is comparable, the solvating power of ammonia and acetonitrile could be compared by reference to their refractive index, respectively 1.32 and 1.34 [13]. The difference between both is very small and could not account for the substantial difference in $\tau_{1}$. The nature of the interaction between the solvating molecule and TDMAE must be invoke, thus implying a much larger interaction with ammonia than with acetonitrile. This is likely indeed since ammonia and not acetonitrile is able to build H-bonds with TDMAE.

To end the discussion, we consider the time constant $\tau_{2}$. In the free TDMAE molecule, it corresponds to the energy transfer from $\mathrm{Z}$ to a charge transfer state CT where the positive charge is delocalized over the four nitrogen atoms. he time constant $\tau_{2}$ is equal to $120 \mathrm{ps}$ in that case [1]. When excited TDMAE is solvated by TDMAE in TDMAE clusters $\tau_{2}$ becomes very large and exceeds 500 ps. Such drastic change of the $\mathrm{Z}$ configuration lifetime was tentitatively assigned to a non local character of the $\mathrm{Z}$ configuration with some delocalisation over several TDMAE molecules constituting the TDMAE cluster [5]. A quantitatively similar observation was made in TDMAE(Ar) $\approx 60$ cluster, but different in the interpretation. A $\tau_{2}$ value much larger than 200 ps was measured, but in a situation where argon atom evaporation proceeds during the $\mathrm{Z}$ to CT energy transfer, thus cooling down the molecule and enhancing the transfer time constant to very large values [4]. In the present case, because the ammonia molecules 
are strongly bonded to the excited TDMAE molecule no such evaporation can occur and the measured $\tau_{2}$ value stays close to the free molecule value. In passing, this is also the indication that the region of coupling between $\mathrm{Z}$ and the final charge transfer state CT is almost not affected by the presence of ammonia. This is not too surprising however since both Z and CT carry a large dipole moment and may solvate similarly, at least in the high energy region where they are coupled. In contrast, the the region of the CT state from where free TDMAE fluoresces seems to be severely affected by surrounding ammonia molecules: whereas the free molecule leads to measurable fluorescence signals [1], no fluorescence signal was observed from the TDMAE $\left(\mathrm{NH}_{3}\right)_{\mathrm{n}}$ clusters. Also, ionisation of the TDMAE molecule, when in the CT state turns out to be impossible whatever the focusing conditions a nanosecond ionisation laser at $532 \mathrm{~nm}$ laser when TDMAE is solvated by ammonia, whereas ionisation of the free molecule is fairly easy. In our opinion this is the indication that the CT state is severely quenched by the ammonia environment, if not transformed chemically. Compared to the $\mathrm{Z}$ configuration where the positive charge is carried by one of the central $\mathrm{C}$ atoms, the CT state has the charge delocalized over the nitrogen atoms of the four dimethylamino groups. A further, at least partial, charge delocalisation over surrounding ammonia molecules might explain the quenching property of ammonia with respect to the CT state of TDMAE.

\section{Acknowledgments}

The authors are happy to thank O. Gobert, P. Meynadier and M. Perdrix, who are responsible for developing, maintaining and running the femtosecond laser facility LUCA (Laser Ultra-Court Accordable) of the CEA, DSM/DRECAM. This work is partly supported by the European Community through the PICNIC network (Product Imaging and Correlation: Non-adiabatic Interactions in Chemistry) under contract number HPRN-CT-2002-00183. 


\section{References}

[1] B. Soep, J. M. Mestdagh, S. Sorgues, and J. P. Visticot, Eur. Phys. J. D 14, 191 (2001).

[2] S. Sorgues, J. M. Mestdagh, J. P. Visticot, and B. Soep, Phys. Rev. Lett. 91, 3001 (2003).

[3] S. Sorgues, J. M. Mestdagh, B. Soep, and J. P. Visticot, Chem. Phys. Lett. 391, $254(2004)$.

[4] S. Sorgues, J.-M. Mestdagh, B. Soep, and J.-P. Visticot, Chem. Phys. 301, 225 (2004).

[5] S. Sorgues, J. M. Mestdagh, E. Gloaguen, M. Heninger, H. Mestdagh, J. P. Visticot, and B. Soep, J. Phys. Chem. A 108, 3884 (2004).

[6] A. J. Merer and R.S. Mulliken, Chem. Rev. 69, 639 (1969).

[7] H. Bouas-Laurent, A. Castellan, J. P. Desvergne, and R. Lapouyade, Chem. Soc. Rev. 30, 248 (2001).

[8] Hyuk Kang, Boyong Jung, and Seong Keun Kim, J. Chem. Phys. 118, 6717 (2003).

[9] K. Winkler, J. R. Lindner, V. Subramaniam, T. M. Jovin, and P. Vohringer, Phys. Chem. Chem. Phys. 4, 1072 (2002).

[10] T. Polivka, D. Zigmantas, J. L. Herek, Z. He, T. Pascher, T. Pullerits, R. J. Cogdell, H. A. Frank, and V. Sundstrom, J. Phys. Chem. B 106, 11016 (2002).

[11] Q. Zhong and A. W. Castleman, Chem. Rev. 100, 4039 (2000).

[12] T. E. Dermota, Q. Zhong, and A. W. Castleman, Chem. Rev. 104, 1861 (2004).

[13] R. C. Weast, M. J. Astle, and W. H. Beyer, Handbook of Chemistry and Physics, 65 th edition 1984-1985., CRC, Boca Raton, Florida, 1984. 